\title{
An Improved Commutative Reversible Watermarking and Encryption for Fingerprint Image
}

\author{
Vaibhav B Joshi · Mehul S Raval
}

Received: date / Accepted: date

\begin{abstract}
A fingerprint system database requires: 1. immunity against offensives like unauthorized data injection, denial of service; 2. privacy protection. Ensuring commutativity between reversible watermarking and encryption concurrently satisfy both requirements. However, reversible watermarking in existing commutative schemes has a security hole. Their fingerprint database can be manipulated by watermark substitution and most significant bit tampering. The proposed method improves reversible watermarking in the commutative scheme. Experimental results shows that the improved scheme is tamper-proof against watermark substitution and most significant bit tampering. It effectively avoids unauthorized data injection and denial of service through database.
\end{abstract}

Keywords Commutative $\cdot$ reversible data hiding · encryption · security

\section{Introduction}

Authentication and identification of people at remote location is a necessity in digital era. A biometric like fingerprint is used to accomplish such a task. There are many applications which requires simultaneous privacy and database protection [17]. E.g. in India fingerprints of one billion+ citizens have been collected under Unique Identification Authority of India (UIDAI) project [30].

Vaibhav B Joshi

Rydot Infotech Ltd, Law Garden, Ahmedabad, India

E-mail: vaibhavj@ussspacetech.com

Mehul S Raval

Pandit Deendayal Petroleum University, Raisan, Gandhinagar, India

Tel.: +91-79-2327 5397

Fax: +91-79-2327 5030

E-mail: mehul.raval@sot.pdpu.ac.in 
The database is accessed remotely to authenticate beneficiaries of the Government schemes and transfer money under direct benefit transfer scheme [6]. Recently a report has been published citing leakage of personal information from UIDAI [1]. Therefore, such database requires both data authentication and privacy protection. Similar requirement exists in managing electronic health records [13]. It has been shown that there is information leakage with single measure like encryption [13] and therefore, multiple measures can improve security.

Like any security system, fingerprint framework is subjected to an attack; which is an attempt to by pass or break the security. It can be circumvented by exploiting an existing vulnerability [25]. E.g. authors [25] identified eight points of attack as shown in Figure 1 . Attacks 1,3,5,6 requires access to internal processing of a system and possess greater threat. The authors in $[5$,

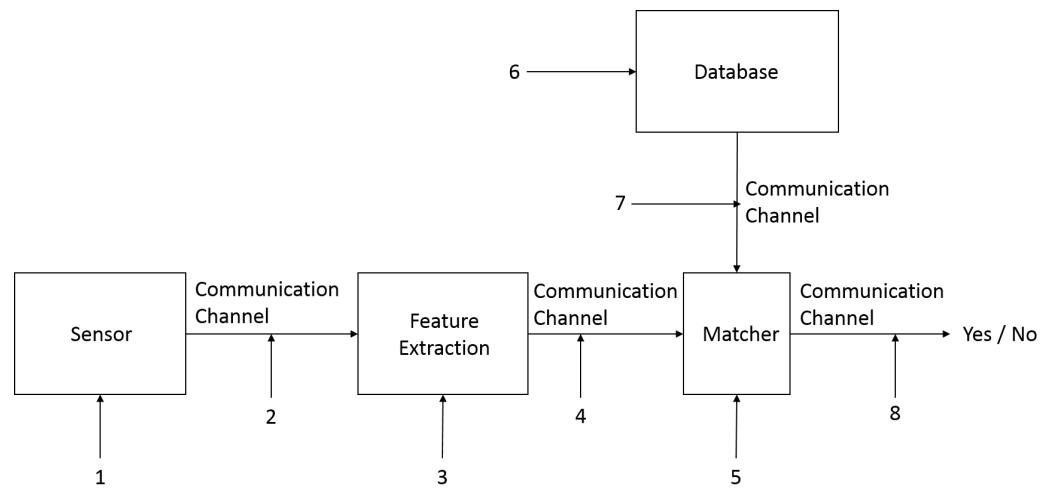

Fig. 1: Eight point of attack model 1

$11,12,27]$ extended the point of attack model and proposed a framework with 20 potential attacks and 22 possible vulnerabilities. Usually an attack is initiated by threat agents [27]. Their goal is to compromise a biometric system to gain unauthorized access. The adversary offensive are categorized as follows [12]: 1. administration or insider; 2. non-secure infrastructure; 3. biometric overtness. The focus of the proposed paper is on an administration offensive. It introduces vulnerability due to loss of integrity during enrollment process, collusion between system admin, user and/or threat agent and misuse of exception handling procedures [12].

An offensive against templates stored in a system database can cause following vulnerabilities [12]: 1 . unauthorized access by placing a fake template; 2. creating a physical spoof from a template [3]; 3. replaying stolen template to the matcher to gain unauthorized access. The database is an easier target as it is clean, de-noised and has a very well defined structure. The templates are also smaller and dataset is also less complex than unprocessed data [27]. 
In case of unauthorized data injection, attacker attempts addition of a unauthorized fingerprint in the database [14]. As seen in Figure 1 (viz. point 6), adversary requires system knowledge for successful data injection. This attack can bypass biometric registration or enrollment phase, allowing positive authentication for an unauthorized fingerprint.

In DoS offensive, threat agent seeks to make a biometric system temporary or permanently inaccessible to the genuine user. In many cases it creates confusion and alarm with real intent to exploit vulnerability in an exception handling process. The execution of the exception program poses a greater threat as: 1 . it is rarely executed; 2 . exhaustive testing for security holes might be missing. There are many ways by which DoS can be launched [12, 27] like physical damage, power supply cutoff, degrade sensor by external factors e.g. temperature, dust, damage data quality by electromagnetic interference, large static electricity, failure to capture and acquire features, administrative abuse like change or damaging the template and triggering false reject. The biometric system also requires robust segmentation mechanism to extract the features $[23,24]$.

\subsection{Background}

The present work is focused on DoS mounted by corrupting fingerprints in a database. It is assumed that threat agents has access to internal system processing and database. Features like minutiae, ridges, delta points are extracted from test and database sample, then they are matched [10] by the fingerprint matcher (viz. Figure 1). It results in the positive authentication if matching score is greater than the set threshold $\tau$. Most Significant Bits (MSBs) of a fingerprint contains large energy and their tampering will not allow correct feature extraction. This yields matching score less than $\tau$ and the system rejects a genuine user. Corrupting many fingerprints in the database by MSB tampering will reduce the system availability to intended users. Unauthorized data injection and DoS are most potent attacks as they compromise the database [27].

One of the defense measure against administration offensive and misuse of exception handling procedures is to build tamper check mechanism [26] in a database [27]. An ideal database protection scheme must have following properties [20]: 1 . template must be unique i.e. it should not match any other in the database; 2 . template must be revocable or cancelable which allows it to be reissued in lieu of a compromised one; 3 . it must be hard to create a spoof from the stolen template; 4 . protection must not degrade recognition performance of the biometric system. A major challenge for database protection scheme is to manage intra user feature variance, i.e. multiple acquisition of the fingerprint does not provide same feature set. This renders storage and comparison of a biometric template in encrypted form (obtained through standards like RSA, AES) futile. 
Reversible data hiding $(\mathrm{RDH})$ has emerged as an alternative authentication mechanism $[21,22]$. When combined with encryption it can provide powerful database protection technique. The important requirements from RDHencryption protection is as follows: 1 . it must satisfy all four properties of ideal database protection scheme; 2 . additionally RDH must tamper-proof database in both plaintext and ciphertext. Using tamper-check in plaintext improves security against an insider offensive. As an alternative it is also possible to decrypt the template and compare it with query template for authentication. Such approach is not secure as the template in plaintext is vulnerable during every authentication attempt. This requires to build tamper-check in ciphertext. One can simultaneously use encryption and reversible watermarking if both operations satisfy commutativity. It has been discussed extensively in [18] with focus on watermarking in an encrypted image. The four properties [18] about commutativity are as follows:

1. The watermarking function can be applied on an encrypted image.

2. The extraction function can reconstruct watermark in the encrypted domain even though it has been embedded in the ciphertext.

3. The extraction function can reconstruct watermark in the encrypted domain even though it has been embedded in the plaintext.

4. The decryption should not change the watermark i.e. affect it's integrity.

Commutative property allows to embed and extract watermark from either a ciphertext or a plaintext as shown in Figure 2. It shows that path number one (dotted lines with arrow $-\rightarrow$ ) is watermarking in ciphertext. The path number two (solid lines with arrow $\rightarrow$ ) is watermarking in plaintext followed by encryption. Here, $\Omega(), E(), K_{E}, K_{W}$ and $W$ represents watermarking function, encryption function, encryption key, watermarking key and watermark respectively. It has been shown in [18] that property number 2 and 3 (or the two paths) are equivalent, if the watermarking $\Omega()$ and encryption function $E()$ commute as shown in Figure 2.

\subsection{Related work}

Commutative schemes are classified in two categories: partial encryption; homomorphic encryption [19]. The former is a simpler approach in which data is divided into mutually exclusive sets. One set is used for encryption and other for watermark embedding. On other hand homomorphic operation describes transformation from one set to another while maintaining relationship among their elements.

In [19] authors incorporate commutativity using discrete cosine transform (DCT). Coefficients are quantized for watermark embedding and their sign is altered during encryption. In [2] tree structured Haar transform is used for encryption and watermark embedding. Authors quantize Haar coefficients and apply bit plane slicing. The sign of all coefficients are stored in binary matrix to form Least Significant Bit (LSBs) of the quantized coefficients. Out of $M$ bits, 


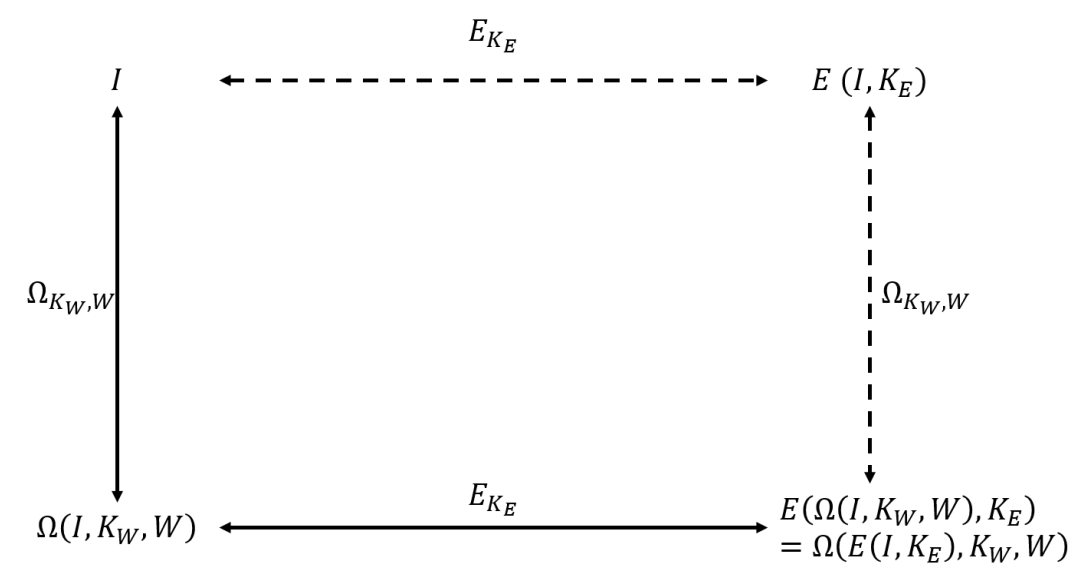

Fig. 2: The functional flow diagram due to commutativity

$K$ most significant bits of quantized coefficients are encrypted using Advance Encryption Standard (AES) and watermark is embedded into remaining $M-K$ bits using scalar quantization. Instead of partial encryption, authors in [28] used permutation based cipher and histogram based additive watermarking. A key based permutation is applied on image pixels which cause loss of perceptual quality. But such permutation does not affect image histogram which is then used for watermarking.

The methods discussed in $[2,19,28]$ ensures commutativity but watermark in them is non-reversible. It means image pixels cannot be recovered exactly after the watermark is removed. Therefore, $[2,19,28]$ cannot be used for authentication in a fingerprint system as non-reversible watermarking will permanently damage fingerprint and it will not satisfy property 4 of an ideal database protection scheme. Therefore, reversibility is a necessity for authentication in a watermarking-encryption framework.

A commutative reversible data hiding and homomorphic encryption scheme has been proposed in [31]. Authors used XOR to incorporate commutativity and lower three bit planes are used to embed watermark. The embedding capacity for an image is calculated using parametric optimization. However, [31] is prone to watermark substitution and MSB tampering. Another commutative encryption - reversible watermarking scheme is proposed in [16].

Commutative schemes [31] [16] have poorly designed reversible watermarking as: 1 . The watermark is independent of the image; 2 . The watermark estimation or copying is easier; 3 . The LSBs fail to register manipulation in MSBs. Also [31] use lower three bit planes for watermarking while [16] use LSBs for watermarking. The above shortcomings increases probability of watermark substitution and MSB tampering for these methods. 


\subsection{Our contribution}

The focus of this paper is to analyze and improve on reversible watermarking in [16]. By strengthening authentication, a fingerprint system becomes more robust against unauthorized data injection and DoS attack due to MSB tampering. Following are merits of the improved method as compared to [31] [16].

- The proposed method extends commutative technique in [16]. It creates two common spaces between watermarking and encryption. One is used for computing watermark from a fingerprint image and other for its embedding. This ensures commutativity along with a image dependent watermark.

- Dependency on fingerprint generates unique watermark. This makes method robust against watermark substitution. It also eliminates a need to store unique watermark for an image. Therefore, the improved method is also free from watermark management issues.

- It detects MSB tampering due to fingerprint based watermark computation and embedding.

- The method has improved security due to its blind nature and larger key space.

- It is using XOR based stream cipher which allows encryption of arbitrary size fingerprint.

\section{Conventional methods}

The proposed method in this paper is an improvement of [16] which is shown in Figure 3. The watermarked and encrypted image is generated by traversing along anyone of the two paths shown in Figure 3. The method maps plain and cipher text such that their LSBs are identical. The common space (LSBs) ensures that $\Omega$ and $E$ are commutative. The methods [31] [16] used two tier arrangement which is dependent on security of each subcomponent. A weakness in anyone can compromise overall security. One way of spoofing authentication system is to carry out watermark substitution [4] attack. The goal by an attacker is to estimate or copy the watermark and insert it into an unauthorized fingerprint. It is then injected into the database. When such an unauthorized sample is presented to the watermark receiver, presence of genuine watermark yields a positive authentication.

The estimation is easy if same mark is used for all fingerprints [4]. A chance of substitution also increase if LSBs are watermarked. In such a case attacker simply replace LSBs of an unauthorized fingerprint with an estimated or copied watermark. Therefore, a successful watermark substitution overrides authentication check making biometric database vulnerable to an unauthorized data injection.

Another way of breaking security is to initiate DoS attack by tampering MSBs of fingerprint in a system database. The watermark based authenti- 


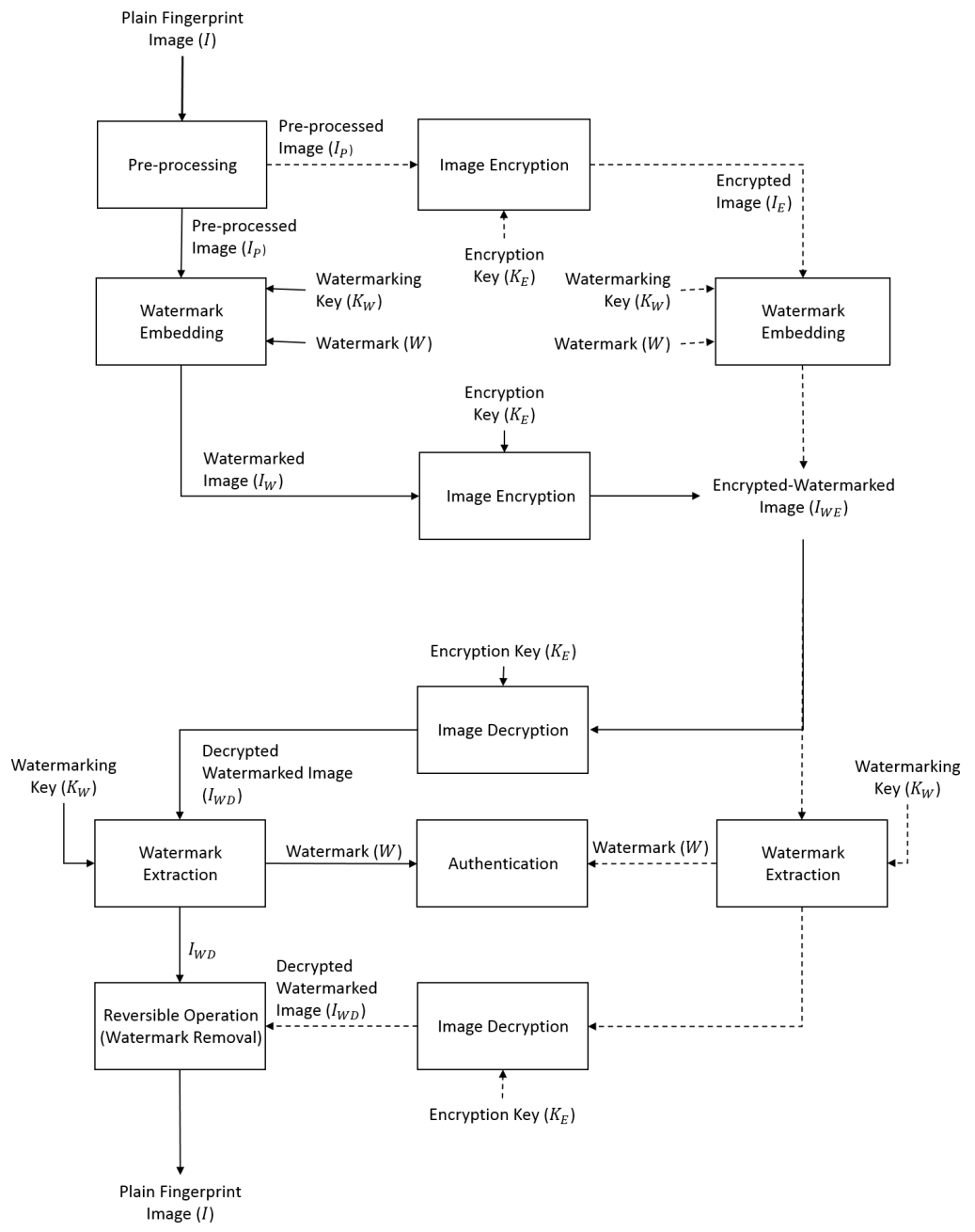

Fig. 3: Basic block diagram showing commutative property between encryption and reversible watermarking. (Arrow type indicate possible watermarking and encryption flows) [16]

cation must detect MSB tampering to flag DoS attack. However, fingerprint independent watermark stored in LSBs fails to register change in its MSBs. The authentication mechanism will find watermark intact and declare fingerprint as authentic for biometric processing. When biometric system extract and compare features from corrupted fingerprint it will generate a false reject. Many false rejects can kick start the exception handling procedure. This is a poorly designed watermarking system as despite of preventive mechanism, DoS can be launched.

The major disadvantages of work in [16] are as follows: 
1. Watermarking method is non-blind in nature i.e. it requires original watermark at the receiver for comparison.

2. The probability of the watermark substitution increases when all fingerprints are marked using the same watermark. This probability can be lowered by using unique watermark for each fingerprint but watermark management becomes a major issue due to big data.

3. The key space is relatively small $\left(2^{8}\right)$ ! and may succumb to brute force search.

\section{The proposed technique}

Following are broad procedural changes in the proposed method as compared to [16]: 1 . watermark computation from the image; 2 . the watermark is embedded in Integer Wavelet Transform (IWT) in place of spatial domain; 3. encryption using robust symmetric key cryptographic technique (XOR) unlike use of a multiplexer; 4 . generation of two common spaces between watermarking and encryption instead of one. Basic flow for the proposed method is shown in Figure 3. The main reason for using IWT is to decompose image in different frequency bands with integer coefficients. This allows application of probability based LSB compression [15] to generate space for watermark embedding.

\subsection{Pre-processing}

Pre-processing creates two common spaces between encryption and watermarking. It has following steps:

1. Take an input image $I$ of size $X \times Y$.

2. Apply integer wavelet transform (IWT) on $I$ to decompose into $L L, L H$, $H L$ and $H H$ bands.

3. Take $H H$ band and randomly permute its LSBs to generate preprocessed band $H H_{P}$.

4. Apply following steps on $L L, L H$ and $H L$ bands to generate preprocessed bands $L L_{P} L H_{P}$ and $H L_{P}$.

(a) Split band into bit planes.

(b) Take least significant bit plane $(L S B P)$ with size $N_{1}$ and divide into non-overlapping blocks of size $2 \times 2$. A row or column ordering on each block yields a bit pattern from 16 possible patterns.

(c) Find the bit pattern (BP) with highest frequency (relative probability) of occurrence.

(d) Apply lossless compression to LSBP using following rules: for each block, if a bit pattern equals BP than corresponding location map bit is set to 1 otherwise it is 0 . Therefore, a location map bit points to a block with or without BP. The size of location map (in bits) for $2 \times 2$ non-overlapping blocks is $25 \%$ of the LSBP.

(e) Generate bit difference by Ex-ORing BP with other patterns. 


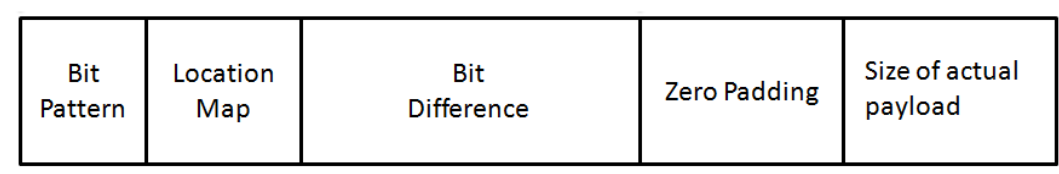

Fig. 4: Fields of reorganized LSB plane

(f) The LSBP is now reorganized in following fields; BP, location map, bit difference, zero padding and size. Let $N_{2}$ be the size of BP, location map and bit difference. The number of zero bits to be padded is $N_{1}$ $N_{2}$ so that size of reorganized LSBP is same as the original one $\left(N_{1}\right)$. The field with zeros is subsequently used for watermark insertion. The reorganized LSBP is shown in Figure 4. The available watermarking capacity in the proposed method is given by size of the zero padding block i.e. $N_{1}-N_{2}$. Table 1 shows difference $N_{1}-N_{2}$ for four sample images and bands $L L, H L$, and $L H$.

(g) All bit planes are recombined to generate the pre-processed band.

5. Take $H H$ band, permute its LSBs using watermarking key $K_{W}$ to generate $H H_{P}$

6. Combine $L L_{P}, H L_{P}, L H_{P}, H H_{P}$ and apply inverse IWT to obtain preprocessed image $I_{P}$.

Table 1: Size of zero padding block $\left(N_{1}-N_{2}\right)$ for sample images

\begin{tabular}{|l|l|l|l|}
\hline Image & $L L$ & $H L$ & $L H$ \\
\hline img1 & 9652 & 2327 & 2172 \\
\hline img2 & 8972 & 2181 & 1958 \\
\hline img3 & 8327 & 2051 & 1937 \\
\hline img4 & 10171 & 2825 & 2341 \\
\hline
\end{tabular}

Start and end positions of the zero padding is known to the watermark embedder and the extractor.

\subsection{Encryption}

A symmetric key cryptography is broadly classified into stream and block cipher. In stream cipher each bit of a plain text is combined with corresponding bit of a key stream i.e. each plain bit is individually encrypted. The proposed technique use stream cipher instead of multiplexer as used in [16] as: 1 . it is easy to implement; 2 . it has maximum entropy because $0 s$ and $1 s$ are generated with equal probability [29]. The steps for the encryption are as follows.

1. As an input use pre-processed $\left(I_{P}\right)$ or watermarked fingerprint image $\left(I_{W}\right)$. 
2. Apply IWT to decompose the input into $L L, L H, H L$ and $H H$ bands.

3. For each band, the following operations are performed to generate encrypted bands $L L_{E}, L H_{E}, H L_{E}$, and $H H_{E}$.

(a) Let $P R N G(K, N)$ be a pseudo random number generator with inputs parameter as key $K$ and range $N$. It generates an outputs which are independent and identically distributed integers from range $[0, N]$. In the proposed work, $N_{\max }$ is the largest coefficient in the input band. Using a secret key $K_{E}$, random numbers $r_{i}(1 \leq i \leq X Y / 4)$ are generated by $\operatorname{PRNG}\left(K_{E},\left\lfloor N_{\max } / 2\right\rfloor\right)$. Finally they are doubled to generate even random integers $2 r_{i}$.

(b) Next XOR even random integer and the input band coefficients to generate encrypted band.

4. Combine the $L L_{E}, H L_{E}, L H_{E}, H H_{E}$ and apply inverse IWT to create encrypted image $I_{E}$.

One must note that band coefficients are XORed with even random integers. It means that coefficient's LSB remains untouched and it can be used for watermark computing as well as embedding. This forms the second common space between watermarking and encryption.

\subsection{Decryption}

1. Take encrypted image $\left(I_{E}\right)$ as the input.

2. Apply IWT to decompose $I_{E}$ into $L L_{E}, L H_{E}, H L_{E}$ and $H H_{E}$ band.

3. For each band, the following operations are performed to obtain the decrypted bands $L L_{D}, L H_{D}, H L_{D}$, and $H H_{D}$.

(a) Use $K_{E}$ to generate even random integers in range [0, $\left.N_{\max }\right]$

(b) XOR random integers and coefficients to generate decrypted band.

4. Use the $L L_{D}, H L_{D}, L H_{D}$ and $H H_{D}$ and apply inverse IWT to obtain decrypted watermarked image $I_{W D}$.

\subsection{Watermark generation}

1. Take input and apply IWT to generate $L L_{X}, L H_{X}, H L_{X}$ and $H H_{X}$ bands. Here $X=P$ for preprocessed image or $X=E$ for encrypted image.

2. Take $H H_{X}$ and extract LSBP.

3. Calculate the message authentication code (MAC) of the LSBP using the cipher block chaining (CBC) mode and AES according to a watermarking key $K_{W}$. The AES-CBC-MAC generates a fixed length of authentication code from an arbitrary length input. The output size depends on the block size of AES which could be 128, 192 and 256 bits. Accordingly the key size is also 128,192 , and 256 bits. Considering watermarking capacity, 256-bit AES seems to be a better choice in the proposed method (cf. Table 1 and section 4.2).

4. The MAC is used as a watermark. 
3.5 Watermark embedding

Divide 256 bits $W_{C}$ into three equal parts i.e. $85+86+85$.

1. Use the encrypted image $I_{E}$ or pre-processed image $I_{P}$ as an input.

2. Apply IWT to generate the $L L_{X}, L H_{X}, H L_{X}$ and $H H_{X}$ band; where, $X=E$ for the encrypted image or $X=P$ for the pre-processed image.

3. For $L L_{X}, H L_{X}$ and $L H_{X}$ band, following operations are performed to generate the watermarked bands, $L L_{W}, H L_{W}, L H_{W}$, respectively.

(a) Separate the band into bit planes.

(b) Replace zero padded block of the LSBP by the watermark to get watermarked LSBP.

(c) Combine the watermarked LSBP with other bit planes to generate the watermarked band.

4. Combine the $L L_{W}, H L_{W}, L H_{W}$ and $H H_{X}$, apply inverse IWT to get watermarked image $I_{W E}$ or $I_{W}$.

\subsection{Watermark extraction and authentication}

Following is the process for watermark extraction.

1. Take the watermarked image $I_{W}$ or $I_{W E}$.

2. Compute watermark $\left(W_{C}\right)$ using key $K_{W}$ as described in section 3.4

3. Apply IWT to generate $L L_{W}, L H_{W}, H L_{W}$ and $H H_{X}$ band. Here $X=P$ or $X=E$.

4. For $L L_{W}, L H_{W}$ and $H L_{W}$ band, following operations are performed to extract watermark.

(a) Separate the band into bit planes.

(b) Extract watermark bits $\left(W_{e x}\right)$ using starting position of the zero padding available from the key $K_{W}$.

5. Concatenate watermark from three bands and generate extracted watermark $W_{e x}$

6. if $W_{C}=W_{e x}$ then the fingerprint is authentic otherwise it is rejected.

\subsection{Reversible operation}

The reversible operation is initiated only if the image is authentic. The steps are as follows.

1. Take the decrypted watermarked image $I_{W D}$.

2. Decompose $I_{W D}$ into four band using IWT.

3. For $L L, L H$, and $H L$ bands, the following operations are performed.

(a) Separate the band into bit planes.

(b) Extract BP, actual payload size and bit difference from LSBP.

(c) Get location map from the LSBP. 
(d) Reconstruct the original LSBP by using BP, location map and bit difference.

(e) Combine all bit planes to generate original band.

4. Take $H H_{P}$ band and apply inverse permutation using $K_{W}$ to its LSBs and get back original $H H$ band.

5. Apply inverse IWT on coefficients of all the bands to get back the image $I$.

In the proposed method watermark is embedded in LSBs of IWT band coefficient. Also when XORed with random even integers keeps these LSB's intact. Thus, watermark remains unaltered in plain or encrypted domain and commutativity is achieved. The $H H$ band is left untouched during pre-processing, watermark embedding or encryption. This facilitates watermark computation in either encrypted or plain domain.

\section{Experimental Results}

4.1 Evaluation

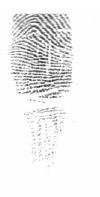

(a)

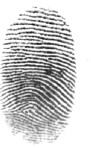

(c)

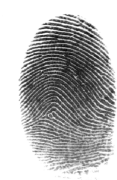

(b)

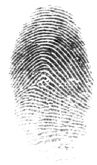

(d)

Fig. 5: Original image, (a) img1 (b) img2, (c) img3 and (d) img4.

The proposed method is evaluated using 50 fingerprint from FVC 2000 [7], FVC 2002 [8] and FVC 2004 [9] databases. Fingerprint images with 500 dots per inch (dpi) and size $X=Y=512$ are selected for experiments. The even random integer during encryption falls in $[0,512)$.

Figure 5 and Figure 6 shows sample of original fingerprints and their watermarked version. In this case all the three encrypted band viz. $L L, H L, L H$ are statistically independent and LSBP of the HH band is also permuted. Figure 7 show encrypted versions of watermarked images. It is clearly visible from 


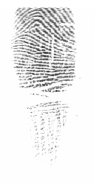

(a)

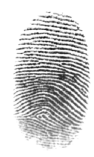

(c)

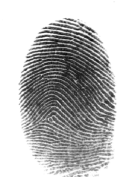

(b)

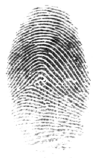

(d)

Fig. 6: Watermarked image, (a)wm_img1 (b) wm_img2 (c) wm_img3 (d) wm_img4.

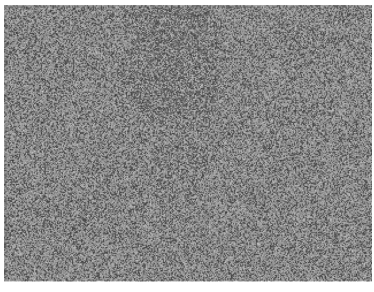

(a)

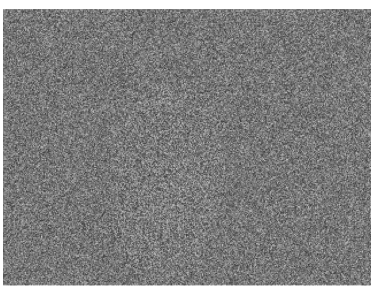

(c)

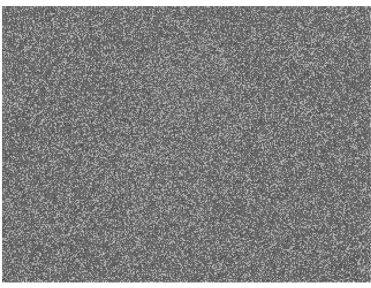

(b)

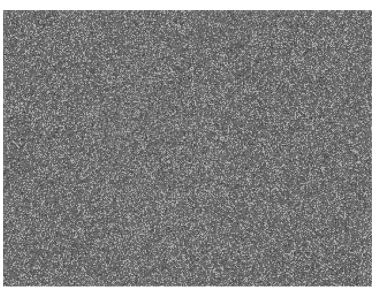

(d)

Fig. 7: Encrypted watermarked image, (a) enc_img1 (b) enc_img2, (c) enc_img3 (d) enc_img4.

Figure 7 that no information about the fingerprint is leaked and encrypted fingerprint cannot be used for authentication. Figure 5 and Figure 6 shows sample of original fingerprints and their watermarked version. In this case all 
the three encrypted band viz. $L L, H L, L H$ are statistically independent and LSBP of the HH band is also permuted. Figure 7 show encrypted versions of watermarked images. It is clearly visible from Figure 7 that no information about the fingerprint is leaked and encrypted fingerprint cannot be used for authentication.

\subsection{Security Analysis}

In the proposed method LSBs of all four bands are left untouched. An attacker knows that watermark is embedded in LSBs of $L L, L H$ and $H L$ bands and computed from the LSBs of $H_{P}$ band. The security of watermark depends on the key $K_{W}$. It can be seen from Table 1 that in the proposed work available embedding capacity across all the three bands $L L, H L, L H$ is significantly larger than 256 bits. Therefore, in the proposed method 256-bit AES is used to generate 256 bits MAC which improves the security in comparison to smaller key size. On average, the Hamming distance for such MAC is 128 bits and therefore success probability that a fake watermark is judged authentic turns out to be $2^{-128}$.

The significance of key $K_{W}$ is empirically demonstrated by computing bit error rate (BER) between the computed watermark from the $H H_{X}$ band (where $X=P$ or $X=E$ ) without key $K_{W}$ and extracted watermark from the LSBs of other three bands. It is used as a metric to compare similarity between computed $W_{C}$ and extracted watermark $W_{e x}$. The BER is computed as follows:

$$
B E R=\frac{D_{H}\left(W_{C}, W_{e x}\right)}{\operatorname{len}\left(W_{C}\right)}
$$

where, $D_{H}(A, B)$ is Hamming distance between two bit strings $A$ and $B$, and $\operatorname{len}(A)$ is the bit length of $A$.

Under any manipulation, statistically number of difference bit in watermarks i.e. $W_{C}$ and $W_{e x}$ is expected to be half of their total bit length. The results are shown in Table 2 . It can be seen that BER is high between the computed and the extracted watermark. Hence without knowing $K_{W}$, it is extremely difficult for malicious parties to create a fake watermark from $\mathrm{HH}$ band.

Table 2: BER between computed watermark without $K_{w}$ and extracted watermark

\begin{tabular}{|l|l|}
\hline Image & BER \\
\hline img1 & 0.6713 \\
\hline img2 & 0.5928 \\
\hline img3 & 0.6159 \\
\hline img4 & 0.6387 \\
\hline
\end{tabular}


The permutation of $H H$ band and use of AES-CBC-MAC as watermark also improves the security.

4.3 Comparison with existing method $[16,31]$

When compared to [16] the proposed method is blind in nature i.e. it does not need original watermark at the receiver for comparison. The watermark is computed and extracted from the watermarked image. Also need to store unique watermark for each fingerprint is eliminated as it is computed from a received image. This reduces storage requirements and brings ease in watermark management. The proposed method is secure against watermark substitution and MSB tampering. The effectiveness of proposed method against these attacks is discussed in following sub-section.

\subsubsection{Watermark substitution}

It is easy to launch watermark substitution attack when LSB plane is used for watermarking. E.g. in [16] LSB contains watermark while in [31] lower three bit planes contains watermark. The attack is mounted by simply replacing the LSBs with watermark bits. Table 3 shows BER of [31],[16] and the proposed method after watermark substitution.

Table 3: BER against watermark substitution

\begin{tabular}{|c|c|c|c|}
\hline Image & {$[31]$} & {$[16]$} & Proposed method \\
\hline img1 & 0 & 0 & 0.3946 \\
\hline img2 & 0 & 0 & 0.3721 \\
\hline img3 & 0 & 0 & 0.4058 \\
\hline img4 & 0 & 0 & 0.4109 \\
\hline Average & 0 & 0 & 0.3958 \\
\hline
\end{tabular}

Table 3 shows the BER against watermark substitution attack i.e. replacing LSBP of fake fingerprint by watermarked LSBP. The BER in both cases [31] and [16] is zero indicating that fake fingerprint has been given positive authentication and an attacker can successfully inject unauthorized data into fingerprint database. On the other hand image dependent watermark in the proposed method captures a mismatch between $W_{C}$ and $W_{e x}$. This secures proposed method against watermark substitution attack.

\subsubsection{MSB tampering}

In this attack, four highest bit planes of watermarked image (they do not contain watermark) are removed (made zero). Such manipulation cause extraction of noisy fingerprint features making DoS successful. Figure 8 shows 
visual effect of MSB tampering on fingerprint images. Table 4 show the BER for [31], [16] and the proposed method against MSB tampering.

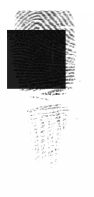

(a)

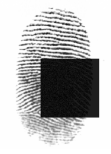

(c)

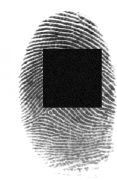

(b)

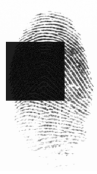

(d)

Fig. 8: Effect of MSB tampering on (a)img1 (b)img2, (c)img3 and (d)img4.

Table 4: BER against MSB tampering

\begin{tabular}{|l|l|l|l|}
\hline Image & {$[31]$} & {$[16]$} & Proposed method \\
\hline img1 & 0 & 0 & 0.4851 \\
\hline img2 & 0 & 0 & 0.4917 \\
\hline img3 & 0 & 0 & 0.4832 \\
\hline img4 & 0 & 0 & 0.4791 \\
\hline Average & 0 & 0 & 0.4847 \\
\hline
\end{tabular}

Tampering of MSBs change fingerprint image characteristics as evident in Figure 8 . However, Table 4 showcase that authentication process in $[16,31]$ fails to detect tampering. In both methods watermark is embedded in LSB and then MSBs are tampered. This does not affect the watermark. Since [16, 31] are non-blind methods, extracted watermark and available watermark match in-spite of the tampering. Thus, the fingerprint clears authentication phase. In the proposed method image dependent watermark captures this tampering as MSBs are also used in watermark computation. The MSB degradation will cause a mismatch between the $W_{C}$ and $W_{e x}$ generating bit error rate (BER). This flags tampering in the fingerprint. The execution of watermark authentication check in off-line mode can capture MSB tampering very early. This precautionary steps can be used to avoid the DoS due to MSB tampering. 


\section{Conclusion}

This work propose a significant improvement in the existing scheme [16]. It satisfies commutative property between encryption and reversible watermarking by using two common spaces to compute and embed watermark. Also due to image dependent watermark, proposed method is secure against watermark substitution and MSB tampering attacks. This provides precautionary steps against unauthorized fingerprint injection and the DoS attack due to false reject. The method is blind in nature and avoid complex watermark management issues. The method is secure due to use of AES-CBC-MAC and encryption. In future, the work can be extended to asymmetric key cryptography and reversible watermarking.

\section{References}

1. Business Standard, Web Team , New Delhi (2017) Aadhaar can be hacked, data leaked, modi government finally admits. URL http://www.business-standard.com/article/economy-policy/ aadhaar-can-be-hacked-data-leaked-modi-government-finally-admits $\backslash$ -117033100359_1.html

2. Cancellaro M, Battisti F, Carli M, Boato G, De Natale FG, Neri A (2011) A commutative digital image watermarking and encryption method in the tree structured haar transform domain. Signal Processing: Image Communication 26(1):1-12

3. Cappelli R, Maio D, Lumini A, Maltoni D (2007) Fingerprint image reconstruction from standard templates. IEEE transactions on pattern analysis and machine intelligence 29(9)

4. Cox I, Miller M, Bloom J (2001) Digital Watermarking. The Morgan Kaufmann Series in Multimedia Information and Systems, Elsevier Science

5. Cukic B, Bartlow N (2005) Biometric system threats and countermeasures: a risk based approach. In: Proceedings of the Biometric Consortium Conference (BCC05)

6. DBT (2013) Direct benefit transfer - dbt. URL https://dbtbharat.gov . in/

7. FVC2000 (2000) Fingerprint database: Fvc2000. URL http://bias .csr . unibo.it/fvc2000/databases.asp

8. FVC2002 (2002) Fingerprint database: Fvc2002. URL http://bias.csr . unibo.it/fvc2002/databases.asp

9. FVC2004 (2004) Fingerprint database: Fvc2004. URL http://bias .csr . unibo.it/fvc2004/databases.asp

10. Jain AK, Prabhakar S, Hong L, Pankanti S (2000) Filterbank-based fingerprint matching. IEEE transactions on Image Processing 9(5):846-859

11. Jain AK, Ross A, Pankanti S (2006) Biometrics: a tool for information security. IEEE transactions on information forensics and security 1(2):125143 
12. Jain AK, Nandakumar K, Nagar A (2008) Biometric template security. EURASIP Journal on advances in signal processing 2008:113

13. Jeremy Kirk, Australia Correspondent, IDG News Service (2015) Even encrypted medical record databases leak information. URL http://www. computerworld.com/article/2980593/data-privacy/ even-encrypted-medical-record-databases-leak-information . html

14. Joshi M, Joshi VB, Raval MS (2013) Multilevel semi-fragile watermarking technique for improving biometric fingerprint system security. In: Intelligent Interactive Technologies and Multimedia, Springer, Berlin, Heidelberg, pp 272-283

15. Joshi MA, Raval MS, Dandawate YH, Joshi KR, Metkar SP (2014) Image and video compression: Fundamentals, techniques and applications

16. Joshi VB, Gupta D, Raval MS (2015) A commutative encryption and reversible watermarking for fingerprint image. In: International Workshop on Digital Watermarking, Springer, pp 323-336

17. Joshi VB, Raval MS, Gupta D, Rege PP, Parulkar SK (2016) A multiple reversible watermarking technique for fingerprint authentication. Multimedia Systems 22(3):367-378

18. Katzenbeisser S, et al. (2005) First summary report on hybrid systems. European ProjectIST-2002-507932, ECRYPT-Network of Excellence in Cryptology, Jan 2005

19. Lian S, Liu Z, Ren Z, Wang H (2007) Commutative encryption and watermarking in video compression. IEEE Transactions on Circuits and Systems for Video Technology 17(6):774-778

20. Maio D, Maltoni D, Jain AK, Prabhakar S (2003) Handbook of fingerprint recognition

21. Ni Z, Shi YQ, Ansari N, Su W, Sun Q, Lin X (2008) Robust lossless image data hiding designed for semi-fragile image authentication. IEEE Transactions on circuits and systems for video technology 18(4):497-509

22. Pankanti S, Yeung MM (1999) Verification watermarks on fingerprint recognition and retrieval. In: Electronic Imaging'99, International Society for Optics and Photonics, pp 66-78

23. Parikh A, Raval MS, Parmar C, Chaudhary S (2016) Disease detection and severity estimation in cotton plant from unconstrained images. In: 2016 IEEE International Conference on Data Science and Advanced Analytics (DSAA), IEEE, pp 594-601

24. Parmar C, Joshi M, Raval MS, Zaveri M (2011) Automatic image inpainting for the facial images of monuments. In: Centenary ConferenceElectrical Engineering, IISc Bangalore, IISc Bangalore, pp 415-420

25. Ratha NK, Connell JH, Bolle RM (2001) Enhancing security and privacy in biometrics-based authentication systems. IBM systems Journal 40(3):614-634

26. Raval MS, Joshi MV, Rege PP, Parulkar S (2011) Image tampering detection using compressive sensing based watermarking scheme. In: National Conference on Machine Vision and Image Processing, IEEE, pp 5-9 
27. Roberts C (2007) Biometric attack vectors and defences. Computers \& Security 26(1):14-25

28. Schmitz R, Li S, Grecos C, Zhang X (2012) A new approach to commutative watermarking-encryption. In: IFIP International Conference on Communications and Multimedia Security, Springer, pp 117-130

29. Tuyls P, Hollmann HD, Lint JV, Tolhuizen L (2005) Xor-based visual cryptography schemes. Designs, Codes and Cryptography 37(1):169-186

30. Unique Identification Authority of India (2017) Uidai. URL https:// uidai.gov.in/

31. Zhang X (2013) Commutative reversible data hiding and encryption. Security and Communication Networks 6(11):1396-1403 